\title{
Single-stranded RNA oligonucleotides that recruit endogenous hnRNPA1 enable the targeted reduction of gene expression
}

\author{
Justin Melendez ${ }^{1}$, Ananya Pal ${ }^{1}$, Sidharth Puram ${ }^{1}$, and Robi Mitra ${ }^{1}$ \\ ${ }^{1}$ Washington University School of Medicine in Saint Louis
}

July 12, 2021

\begin{abstract}
Targeted gene knockdown has become one of the most powerful tools in molecular biology and holds substantial promise in therapeutic applications. While existing technologies such as siRNAs, CRISPRi, and ASOs effectively and specifically reduce gene expression, few can be used to first discover the genes that influence a particular phenotype and then directly transition to being used as oligonucleotide therapeutics. Thus, a tool that could help bridge the gap between target discovery and the development of therapeutic leads would benefit the scientific community. Here, we present hnRNPA1 recruiting oligonucleotides, or AROs, as single-stranded RNA (ssRNA) molecules that knockdown transcript levels of target genes. AROs target specific pre-mRNA transcripts via sequence homology and leverage the ubiquitous and abundant endogenous RNA-binding protein hnRNPA1 to degrade target transcripts. Using RT-qPCR, we show that AROs effectively knock down target genes when delivered via a plasmid and expressed using a Pol II promoter or when delivered directly as single-strand RNAs. Additionally, as proof of principle, we use a ssRNA ARO to knockdown KRT14 in squamous cell carcinoma and show reduced invasive potential. We believe AROs fill an important niche in the scientific toolbox by taking advantage of endogenous RNA binding machinery for RNA knockdowns.
\end{abstract}

Single-stranded RNA oligonucleotides that recruit endogenous hnRNPA1 enable the targeted reduction of gene expression

\section{AUTHORSHIP}

Justin Melendez ${ }^{1}$, Ananya $\mathrm{Pal}^{2}$, Sidharth V. Puram ${ }^{2}$ Robi D Mitra ${ }^{1^{*}}$.

${ }^{1}$ Department of Genetics and Center for Genome Sciences and Systems Biology, Washington University in St. Louis, St. Louis MO, 63110

${ }^{2}$ Department of Otolaryngology - Head and Neck Surgery and Department of Genetics, Washington University in St. Louis, St. Louis MO, 63110

* Correspondence to: rmitra@wustl.edu

\section{GRANTS}

This work was supported by NIH Grants RF1MH117070, RF1MH117070-S1, U01MH109133 (National Institute of Mental Health) (R.D.M., J.M), R01GM123203 (National Institute of General Medical Sciences) (R.D.M.).

\section{ABSTRACT}

Targeted gene knockdown has become one of the most powerful tools in molecular biology and holds substantial promise in therapeutic applications. While existing technologies such as siRNAs, CRISPRi, and ASOs effectively and specifically reduce gene expression, few can be used to first discover the genes that influence 
a particular phenotype and then directly transition to being used as oligonucleotide therapeutics. Thus, a tool that could help bridge the gap between target discovery and the development of therapeutic leads would benefit the scientific community. Here, we present hnRNPA1 recruiting oligonucleotides, or AROs, as single-stranded RNA (ssRNA) molecules that knockdown transcript levels of target genes. AROs target specific pre-mRNA transcripts via sequence homology and leverage the ubiquitous and abundant endogenous RNA-binding protein hnRNPA1 to degrade target transcripts. Using RT-qPCR, we show that AROs effectively knock down target genes when delivered via a plasmid and expressed using a Pol II promoter or when delivered directly as single-strand RNAs. Additionally, as proof of principle, we use a ssRNA ARO to knockdown KRT14 in squamous cell carcinoma and show reduced invasive potential. We believe AROs fill an important niche in the scientific toolbox by taking advantage of endogenous RNA binding machinery for RNA knockdowns.

\section{KEYWORDS}

Gene knockdown, RNA therapy, Synthetic biology, hnRNPA1, Splicing regulatory element, Epithelialmesenchymal transition, KRT14.

\section{INTRODUCTION}

The ability to manipulate RNA expression and splicing is one of the most powerful tools in research and holds great promise for therapeutic applications. In basic science, the ability to target and knockdown a specific gene of interest using tools such as siRNA and CRISPRi has allowed us to unravel the intricacies of biological pathways, leading to many new discoveries (Cong et al., 2013; Gilbert et al., 2014; Kampmann, 2018; Mullenders et al., 2009). In medicine, targeted manipulation of expression and splicing through antisense oligonucleotides (ASOs) has led to several promising therapeutics. For instance, the FDA has approved ASObased treatments for both Duchenne muscular dystrophy, a child-onset disease in which patients progressively lose muscle function resulting in wheelchair dependency, the need for ventilation assistance and ultimately premature death (Aartsma-Rus \& Krieg, 2017), and spinal muscular atrophy in infants, a disease caused by mutations in SNM1 leading to musculoskeletal wasting and respiratory failure (Stein \& Castanotto, 2017).

While a variety of technologies exist to knockdown or modify pre-mRNA in the cell, few options are available that bridge the gap between research and clinical settings. Tools such as siRNA, lentivirus, and plasmid expression are frequently employed as screening tools to find therapeutic targets (Koike-Yusa et al., 2014). However, the end products of these methods often run into roadblocks as therapeutics themselves. siRNA therapies are challenged by double-stranded RNA degradation by the immune system, off-target effects due to improper strand loading, and toxicity due to oversaturation of the RNAi machinery (Nogrady, 2019). CRISPR-based technologies require the introduction of the exogenous Cas9 protein, which poses a significant barrier for many applications, and their off-target effects are still being evaluated for safety (Tycko et al., 2019). These limitations result in the identification and characterization of gene targets with technologies that are either clinically unviable or suboptimal, thus requiring their re-evaluation with small molecule screens or other therapeutic methods, resulting in significant delays in translating bench observations to the clinical setting.

While ASOs have found purchase as therapeutics, they are heavily reliant on a variety of nucleotide chemistries and backbone modifications to achieve the desired results (Roberts et al., 2020). ASOs that degrade their targets are often composed of RNA with central DNA "gap" sequences that hybridize with homologous regions of RNAs and trigger RNase $\mathrm{H}$ mediated degradation, whereas sterically hindering ASOs, such as morpholinos, incorporate morpholine moieties instead of ribose backbones and modified phosphonodiamidite linkages to evade degradation (Ekker, 2006). Reliance on these chemistries and modifications makes it difficult to perform large ASO based screens to identify genes that can be targeted with this modality. For example, this methodology is incompatible with the production of lentiviral libraries, which infect cells clonally and thereby can greatly parallelize the search for candidate molecules through a combination of cell culture and next-generation sequencing.

A technology that could seamlessly transition between construct-based screening and the synthesis of bio- 
logically functional molecules would greatly benefit both the research and clinical communities. The ideal technology would require minimal chemical modifications to transition from screening construct to lead compound, be compatible with modern molecular biology techniques, such as plasmid or viral expression, and would not require exogenous proteins to function. To address this need, we created hnRNPA1 recruiting oligonucleotides (AROs) to serve as both a standalone technology and paradigm for using endogenous RNA binding proteins to manipulate pre-mRNA.

hnRNPA1 is a ubiquitously expressed RNA binding protein with multiple roles that are still being uncovered; however, the protein is best known for the role it plays in alternative splicing and RNA processing (JeanPhilippe et al., 2013). Splice sites are defined by short 5' (GU) and 3' (AG) sequences flanking introns. While these dinucleotides are required for splicing, they do not contain enough information to correctly specify splice junctions. As a result, regulatory proteins such as SR proteins and heterogeneous nuclear ribonucleoproteins (hnRNPs) must bind to exonic splice enhancers (ESEs) and exonic splice silencers (ESSs) respectively to properly define exon-intron boundaries (Lee \& Rio, 2015) . Binding of hnRNPA1 to an ESS on pre-mRNA blocks the binding of SR proteins and splicing machinery leading to cooperative recruitment of additional hnRNPA1 and suppression of the splice site (Jean-Philippe et al., 2013).

We sought to harness the ability of hnRNPA1 to suppress splice site selection by recruiting it to targeted pre-mRNA molecules. To do so, we constructed single-strand RNA molecules herein referred to as hnRNPA1 recruiting oligonucleotides or "AROs" (for $A 1 r$ ecruitingo ligonucleotide). AROs consist of two parts, a short (20 - $25 \mathrm{bp})$ RNA oligonucleotide targeting domain which is complementary to the target pre-mRNA, and an hnRNPA1 recruiting loop derived from the HIV ESS 3, which binds to the RNA binding domain RRM1 of hnRNPA1 and recruits the protein (Jain et al., 2017) (Figure 1A). We hypothesized that, upon recruitment to the target pre-mRNA, hnRNPA1 would displace local SR proteins and splicing machinery (Figure 1B) resulting in the suppression of regional splice sites, leading to frameshifts caused by aberrant exon skipping or intron inclusion.

Due to the ubiquitous nature of endogenous hnRNPA1 across cell types, AROs do not require any exogenous proteins to function. Further, as AROs do not rely on DNA-RNA hybrids to trigger RNAase H degradation, AROs can be produced entirely as RNAs within the cell. Finally, their single-stranded nature and simple mechanism of action allow them to be transcribed in vivo using a standard Pol II promotor, so they are compatible with standard lentiviral screening methodologies. In the following experiments, we demonstrate that AROs can suppress target mRNA transcripts, are biologically functional, and can be expressed using standard molecular biology constructs or delivered directly as single-strand RNA oligonucleotides.

\section{RESULTS}

\section{AROs knock down KRT14 expression in an hnRNPA1 specific manner.}

To test whether AROs can knock down gene expression in a targeted manner, we created a 23 base-pair antisense RNA molecule with homology to exon 7 of the KRT14 gene and fused an hnRNPA1 recruiting loop to either the 5' end, the 3' end or both sides of the targeting oligonucleotide. KRT14 was chosen as a knockdown target because it has been implicated as a driver of the epithelial-mesenchymal transition in numerous cancers, (Bilandzic et al., 2019, p. 14; Papafotiou et al., 2016, p. 14) and Exon 7 was chosen due to its small size (47 base-pairs) and because it contains several motifs for ASF/SF2 exonic splice enhancer binding, a regulatory element that hnRNPA1 has been shown to repress (Cartegni et al., 2003; Kashima et al., 2007). As controls, we also tested an oligonucleotide containing only the $23 \mathrm{bp}$ targeting domain (i.e. an "antisense only" control with no A1 recruiting loop) and an ARO with a scrambled targeting domain (Fig 2A), which respectively served to determine whether the targeting oligonucleotide or hnRNPA1-recruiting loop alone influenced KRT14 expression. Human keratinocytes, HaCat cells, were chosen as a model cell line for initial experiments due to their robust expression of KRT14.

Because hnRNPA1 binds cooperatively (Jean-Philippe et al., 2013), we hypothesized that we would see the greatest knockdown of gene expression with the ARO containing two flanking hnRNPA1 recruiting loops (ARL-Oligo-ARL). Consistent with our expectations, we observed a 56\% knockdown of KRT14 gene 
expression in ARL-Oligo-ARL compared to the carrier control $(\mathrm{P}<=0.001)$, and a $53 \%$ knockdown of expression compared with the targeting oligo only (Oligo) control $(\mathrm{P}<=0.01)$ indicating that the hnRNPA1 recruiting loop was required for knockdown to occur (Figure 2B). We also saw significant knockdown of the ARL-Oligo $(\mathrm{P}<=0.05)$ and Oligo-ARL $(\mathrm{P}<=0.05)$ experimental conditions (18\% and $29 \%$ respectively), when compared to the targeting oligo only (Oligo) control. Interestingly, we observed a small (15\%) but statistically significant difference between the targeting oligo only control (Oligo) and the scrambled targeting oligo control attached to an hnRNPA1 recruiting loop (scrOligo-ARL) indicating that the presence of the ARL alone may have a minor knockdown effect on KRT14. Alternatively, it is possible that the scrambled targeting oligo has off-target effects influencing the expression of other transcripts which affect the expression of KRT14.

To test whether the effects of the AROs were hnRNPA1 dependent, each condition was repeated in the presence of two validated siRNAs designed to knockdown hnRNPA1 (Figure 2B). Concurrent loss of hnRNPA1 with expression of ARL-Oligo, Oligo-ARL, and ARL-Oligo-ARL caused significant and complete loss of previously seen knockdown effects on KRT14 transcript $(\mathrm{P}<=0.05, \mathrm{P}<=0.01$ and $\mathrm{P}<=0.01$ respectively). In contrast, differences between the control constructs, scrOligo-ARL and Oligo, and their hnRNPA1 siRNA knocked down counterparts were not significant.

Taken together, our results demonstrate that AROs targeting KRT14 knock down mRNA expression in a targeted and hnRNPA1 dependent manner.

\section{AROs targeted to TBK1 knock down RNA expression across multiple locations on exon 14.}

To evaluate whether AROs could knock down the expression of other genes, we designed AROs against the TBK1 gene (Figure 3A). We targeted exon 14 because it contained multiple ASF/SF2 sites according to ESE finder (Cartegni et al., 2003) and because its moderate size (122 bp) allowed us to evaluate multiple AROs targeting the same exon, as diagrammed in Figure 3A. To investigate whether AROs act in a location-specific manner, we designed 5 different AROs targeting exon 14 of TBK1 in 20 base pair segments and tested them in HaCat cells. All 5 AROs significantly knocked down TBK1 expression $(\mathrm{P}=<0.01)$ when compared to the mock transfection carrier control. The largest knockdown was seen in ARL-TBK1-O3-ARL (51\%) and the smallest in TBK1-O5-ARL (37\%). These results indicate that all AROs targeting exon 14 of TKB1 were effective at gene knockdown.

As a negative control, we included the ALR-Oligo-ARL construct targeting the KRT14 gene from the previous experiment. As expected, the KRT14-targeting ARO did not decrease TBK1 transcript levels. Instead, we observed a significant $(\mathrm{P}<=0.01)$ increase in TBK1 gene expression when KRT14 is knocked down. We hypothesize that the increase in TBK1 expression is a downstream result of perturbation of KRT14 expression levels.

Our experiments do not seem to indicate any preference for targeting oligo location along the length of the exon as all of the oligonucleotides that targeted exon 14 of TBK1 showed similar knockdown effects.

\section{AROs can be delivered directly via lipofection as synthesized oligonucleotides.}

A major appeal of AROs as a potential therapeutic is their small size and single-stranded nature. Smaller molecules are easier to deliver and single-stranded RNA is not targeted by cellular mechanisms that actively seek out double-stranded RNA for degradation. To determine if AROs could be delivered directly as singlestranded RNA oligonucleotides, we ordered synthesized AROs targeting KRT14 and tested them in HaCat cells. Each molecule was designed to contain phosphorothioate bonds between the first two and last two bases to inhibit exonuclease degradation. The targeting domain was the same sequence used in the previous plasmid based knockdown experiment (Figure 1). We tested a single 5' ARL (ARL-Oligo) as well as ARLs fused to each end of the targeting oligo (ARL-Oligo-ARL) and compared them to a previously validated siRNA targeting KRT14 at varying concentrations (Figure 4A).

We observed significant knockdown effects at all concentrations of ARL-Oligo and anti-KRT14 siRNA conditions, with diminishing returns at above 100nM. Interestingly, we did not observe significant knockdown 
with ARL-Oligo-ARL oligos, despite this configuration being the most effective in plasmid experiments, until concentrations of $1 \mu \mathrm{M}$. One possibility for this may be that the ARL-Oligo-ARL may have had difficulty making it to the nucleus due to its increased size. Another possibility is that interactions between complementary regions in the two ARLs on the oligo caused the targeting domain to be less available or degraded more quickly than its single ARL counterpart.

\section{Knocking down KRT14 via AROs in squamous cell carcinoma reduces cancer migration capa- bility.}

Having demonstrated that we can knock down genes via delivery of oligonucleotide AROs, we next sought to test if ARO-mediated gene knockdown could achieve a therapeutic/biologically functional effect. KRT14 expression has been shown to be important for metastasis and invasion in several cancers including bladder and ovarian cancers (Bilandzic et al., 2019, p. 14; Papafotiou et al., 2016, p. 14). SCC9 is a squamous cell carcinoma line used as a model for epithelial-mesenchymal transition studies in head and neck cancer (Zhu et al., 2012). We hypothesized that knocking down KRT14 in SCC9 using our ssRNA anti-KRT14 ARO would reduce the ability of these cells to invade surrounding tissues.

To test this hypothesis, we lipofected SCC9 cells with a KRT14 targeting ARO (ARL-Oligo) or, as a positive control, an siRNA against KRT-14, at $100 \mathrm{nM}$ and assayed RNA expression by RT-qPCR. After 48 hours a near complete knockdown of KRT14 transcript levels was observed in both conditions $(\mathrm{P}<=.001)$ (Figure 4B). We also sought to verify knockdown of Cytokeratin-14, the protein encoded by KRT14. We performed a western blot (Figure 5C) and observed a $38 \%$ reduction of protein in the siRNA treated cells $(\mathrm{P}<=0.01)$ and a $24 \%$ knockdown in cells treated with ssRNA anti-KRT14 ARO $(\mathrm{P}<=0.05)$. While this knockdown was less than the observed RNA reduction, this is likely due to two factors: the long doubling time of SCC9 ( 72 hours) contributing to slow dilution of the protein, and the protein turning over at a slower rate than the RNA, which is actively being degraded in both conditions. Additionally, we observed that differences in protein knockdown between the ARO and siRNA were not significant.

We next sought to assay the invasive potential of SCC9 cells after the loss of KRT14. 48 hours after lipofection with synthesized oligonucleotides or siRNAs, cells were seeded into a Boyden chamber to test their ability to invade into surrounding tissues. This assay uses a transwell that is sealed on one side with a porous membrane coated with Matrigel. The transwell is then suspended inside a larger well, and only invasive cells are able to migrate through the Matrigel. 48 hours after transfection, cells were seeded and incubated for 24 hours. Cells that invaded the Matrigel were then stained, observed via light microscopy (Figure 5B), and counted. We observed a significant decrease in invasive potential of cells treated with both the anti-KRT14 siRNA control $(\mathrm{P}<=0.05)$ as well as our ARO $(\mathrm{P}<=0.05)$ compared to cells treated only with lipofectamine carrier. Cells treated with the KRT14 siRNA had 39\% fewer cells migrate into matrigel than the carrier control, while cells treated with the anti-KRT14 ARO had $43 \%$ fewer cells relative to the carrier (Figure 5A).

The above results support two important conclusions. First that the knockdown of KRT14 negatively affects the ability of squamous cell carcinoma to invade surrounding tissues. Second that AROs delivered as ssRNAs are biologically functional and able to knock down protein levels in addition to RNA. We believe these experiments show the usefulness of AROs as novel and useful biological tools for both RNA and protein perturbation.

\section{DISCUSSION}

In this article, we present hnRNPA1 recruiting oligonucleotides (AROs), a new tool for mRNA knockdown consisting of a short targeting oligonucleotide and a hnRNPA1 recruiting loop. Through a series of RT-qPCR experiments targeting KRT14 and TBK1, we have shown that AROs expressed from plasmids are able to knock down the expression of two independent genes in a targeted fashion. We have shown that both the antisense targeting RNA and the hnRNPA1 recruiting loop must be present for an efficient knockdown. We have also shown that AROs function in an hnRNPA1 dependent manner. Finally, we have shown that AROs are functional in multiple cell lines including human keratinocytes (HaCat) and squamous cell carcinoma 
cells (SCC9) and can affect a loss-of-expression functional phenotype.

Our results suggest that the location of the sequence targeted by an ARO on the exon of interest does not have a large influence on knockdown efficiency. We initially hypothesized that the targeting location would have a bigger impact on ARO function. Specifically, we expected that it would be necessary to direct AROs to splice junctions or to ASF/SF2 motifs, as preventing binding of SR splice enhancer proteins is a known mechanism of hnRNPA1 splice site repression (Jean-Philippe et al., 2013; Kashima et al., 2007). One possible explanation is the cooperative recruitment of hnRNPA1 molecules along RNA in the 3' to 5' direction (Jean-Philippe et al., 2013). This might mean that an ARO binding at a downstream exonic sequence is sufficient to suppress SR protein recruitment at upstream regions. Another possibility is that the exon we choose was too small to see location specific effects. Larger exons with more space between splice sites should be tested in the future to help answer this question.

A major advantage of AROs over most knockdown technologies is the ease with which they can transition from promotor-based expression (i.e. from a plasmid or lentivirus construct) to RNA oligonucleotides that can be directly delivered to cells or organisms. Here we have shown the utility of AROs as a potential therapeutic by synthesizing single-stranded RNA AROs and showing that they are able to achieve similar levels of knockdown as their plasmid counterparts. By using ssRNA AROs to knock down KRT14 in SCC9, a squamous cell carcinoma line used as a model for epithelial-mesenchymal transition studies in head and neck cancer, we demonstrate that ARO-based knockdown resulted in a biologically relevant phenotype - the decreased ability of carcinoma cells to migrate in a cell invasion assay.

An unexpected finding in our RNA oligonucleotide experiments was that while the double ARL ssRNA (ARL-Oligo-ARL) worked to some degree at high concentrations, the single ARL (ARL-Oligo) achieved significantly better gene knockdown at lower concentrations. This contrasted with our plasmid experiments which found that double ARL AROs performed best. A possible explanation for this may be that the additional size or complexity of double ARL oligonucleotides may have lessened their ability to make it to the nucleus. Another possibility is that complementary regions between the two ARLs caused secondary structure leading to double-stranded portions of the construct being degraded in the cytoplasm. This would be less likely to occur in the plasmid construct as those AROs would be produced in the nucleus and would presumably have more opportunity to affect pre-mRNA before being exported and degraded.

We hypothesize that AROs knock down gene expression by causing errors in pre-mRNA splicing followed by nonsense-mediated decay. However, it is important to point out that there are several plausible alternative hypotheses. For example, hnRNPA1 has been shown to bind to the 3' UTR of some genes, and this can lead to mRNA deadenylation and degradation (Geissler et al., 2016). This may be why binding along any point of an exon in pre-RNA seems to be effective. While more work will need to be done to determine the specific mechanism of action of AROs to better understand and improve their function, their utility as biological tools in their current form remains substantial.

AROs represent a novel paradigm for recruiting RNA binding proteins to pre-mRNAs via synthetic constructs. Here we have shown that hnRNPA1 can be recruited via a short targeting oligonucleotide to knock down transcript levels of a target gene. While more work will need to be done to understand the exact mechanism of how AROs function, the work here illustrates their potential as a novel, biologically functional way to knock down target transcripts through the redirection of endogenous cellular machinery. Because RNA binding proteins are ubiquitous and abundant across cell types, their recruitment to targeted pre-mRNAs may be a useful way to modulate RNA production, modification, or degradation. The diverse array of functions they perform and their directable nature make them an ideal source to mine for molecular biology tools. The ability to express AROs, and similarly designed molecules, from a Pol II promoter also has substantial advantages. AROs could be placed under the control of cell or tissue specific promotors and used in tissue specific studies or placed behind activatable promotors.

AROs can both be expressed by constructs or used directly as ssRNA molecules with little modification. This dual functionality puts them in a unique position to bridge the gap from high-throughput screens to 
independently functional molecules. We believe AROs fill an important niche in the scientific toolbox and we hope the work here can serve as a template for future constructs that make use of the potential of RNA binding proteins as tools for the modulation of RNA homeostasis.

\section{MATERIALS AND METHODS}

\section{Cell culture}

HaCat cells were cultured in Dulbecco's Modified Eagle Medium (Gibco) with 10\% FBS at 37 degrees Celsius at 5\% CO2 and maintained in flat bottom T75 flasks. SCC9 cells were cultured in a 3:1 mixture of Ham's F12 Medium (Gibco) and Dulbecco's Modified Eagle Medium supplemented with 10\% FBS and maintained at 37 degrees Celsius at 5\% CO2 in T75 flasks. For ARO RT-qPCR experiments, cells were grown in 24 well plates and lipofected with $500 \mathrm{ng}$ of plasmid using lipofectamine 3000 (Thermofisher). In knockdown experiments, two independent siRNAs (Life Technologies, Silencer Select Pre-Designed siRNA) were co-transfected at a final concentration of $10 \mathrm{nM}$ during lipofection, and knockdown of the target gene was verified via RT-qPCR. In experiments involving direct delivery of single-stranded RNA, cells were grown in 24 well plates and lipofected using lipofectamine 3000 (Thermofisher) at the concentrations listed. For Western blot and Boyden chamber experiments, cells were maintained in 6 well plates prior to seeding.

\section{Boyden Chamber Invasion Assays}

20,000 SCC9 cells were suspended in 200 ul of serum-free growth media and seeded inside a matrigel-coated transwell. $750 \mathrm{ul}$ of growth media containing $10 \%$ FBS was added to the larger well. Cells were allowed to migrate through the pores to the other side of the membrane by incubating them at 37 degrees Celsius for 24 hours. After 24 hours of incubation, the transwells were washed in PBS, fixed in 100\% Methanol for 2 minutes, and then stained in crystal violet solution for 20 minutes. Excess crystal violet was washed off by dipping the transwells in water. The transwells were then left to dry following which they were imaged in a brightfield microscope. A custom Image J script was used to count cells that migrated into the Matrigel coated side of the transwell.

\section{Western Blots}

Cells were harvested 48 hours after lipofection using RIPA buffer and total cellular protein was extracted. A BCA assay (Pierce BCA Protein Assay Kit) was used to determine the protein concentration of each sample. $5 \mathrm{ug}$ of lysate from each sample was loaded into a Mini-PROTEAN TGX stain-free precast gel (Bio-Rad) and resolved. Once proteins were resolved, gels were activated on a ChemiDoc (Bio-Rad) imager and were then transferred to a polyvinylidene difluoride (PVDF) membrane. Membranes were probed with mouse Cytokeratin 14 antibody primary antibody (Life Technologies) and HRP conjugated goat anti-mouse secondary antibody (Santa Cruz Biotechnology). Total proteins and chemiluminescence was imaged on the ChemiDoc and Image Lab Software (Bio-Rad) was used to analyze protein abundance.

\section{Statistical analysis}

A Student's t-test was used to test for significance between groups. Experiments were performed in at least two replicates unless otherwise stated and error bars show plus and minus standard deviation. $\mathrm{P}$ values of $<=0.05$ were determined to be significant.

\section{RT-qPCR}

Cells were grown for 48 hours after lipofection before being harvested using RNAprotect (Qiagen). Total RNA was extracted using Direct-zol RNA microprep kit and quantified. Each condition was performed in triplicate using $50 \mathrm{ng}$ of total RNA. For each $20 \mathrm{ul}$ reaction, RNA was added to Luna Universal One-Step RT-qPCR master mix (New England Bio Labs) and two RT-qPCR primers at a final concentration of $0.4 \mu \mathrm{M}$. The following amplification cycle was run on a QuantStudio 3 realtime PCR machine (ThermoFisher Scientific): $55{ }^{\circ} \mathrm{C}$ for $10 \mathrm{~min}, 95{ }^{\circ} \mathrm{C}$ for $1 \mathrm{~min}$, followed by 95 ${ }^{\circ} \mathrm{C}$ for $10 \mathrm{sec}$ and $60{ }^{\circ} \mathrm{C}$ for $1 \mathrm{~min}$ for 40 cycles. All genes were normalized to beta Actin (ACTB) and expression was determined via relative quantification to lipofectamine only carrier control. Gene 
specific primers for each gene of interest were ordered from Integrated DNA Technologies and are as follows: ACTB: 5'-CACCATTGGCAATGAGCGGTTC-3' and 5'-AGGTCTTTGCGGATGTCCACGT-3', TBK1: 5'-GGATCACTGCCATTTAGACCC-3' and 5'-CAGGCATGTCTCCACTCCAG-3' (PrimerBank 309747068c3), KRT14: 5'- TGAGCCGCATTCTGAACGAG-3' and 5'-GATGACTGCGATCCAGAGGA3' (PrimerBank 83641893c1), HNRNPA1: 5'-TCAGAGTCTCCTAAAGAGCCC-3' and 5ACCTTGTGTGGCCTTGCAT-3' (PrimerBank 83641893c1).

\section{Plasmids and Cloning}

A sequence containing two BSMBI restriction sites flanked by hnRNPA1 recruiting loops (ARLs) was cloned into PCDNA 3.1+ (Invitrogen) behind the CMV promotor between NheI and XbaI restriction sites, upstream of the polyadenylation sequence to create a Golden Gate vector for ARO constructs. This vector was then used to clone all additional ARO constructs except for scrOligo-ARL, Oligo, ARL-Oligo, and Oligo-ARL (Figure 2A) which were cloned into the same locus via Gibson assembly. All Golden Gate cloned constructs were ordered as two single-strand DNA oligos (Integrated DNA Technologies) and annealed such that overhangs were compatible with the Golden Gate ARO vector. Once cloned, these annealed oligos served as the targeting oligos for the AROs. Single-strand RNA ARO oligos were ordered directly from IDT (Integrated DNA Technologies) and contained two phosphorothioate bonds at each end to protect the RNA from degradation by exonucleases. The sequence used for the ARL was as follows: 5'GGATCCATTCGTAGGAGGAACGGATCC-3'. Sequences for targeting oligos in AROs used in experiments are as follows. ARL-Oligo-KRT14 -ARL: 5'CCAGAGGAGAACTGGGAGGAGG-3', scrambledARL-Oligo: 5'-TGTGGCGAGTAGACTCGAAG-3', ARL-TBK1-O1-ARL: 5'-GTTCCCTGAGAACTGGAAAG3', ARL-TBK1-O2-ARL: 5'-CCTGAAGACTGGTTTCTATT-3', ARL-TBK1-O3-ARL: 5'TCTGCCAGTGATCCACCTGG-3'， ARL-TBK1-O4-ARL: 5'- CTTCTTGATGTGCCCATGCG-3', ARL-TBK1-O6-ARL: 5'- TCTGTCTTTCGGATGAGTGC-3'.

\section{AUTHOR CONTRIBUTIONS}

Justin Melendez conceived of the project and performed all lipofections, cell culture, RT-qPCR, Westernblots, and data analysis and wrote the initial draft of the manuscript. Sid Puram designed and interpreted and Ananya Pal designed, performed, and interpreted the Boyden chamber assays and both reviewed the manuscript. Rob Mitra contributed to the direction of the project and reviewed the manuscript.

\section{ACKNOWLEDGMENTS}

This work was supported by NIH Grants RF1MH117070, RF1MH117070-S1, U01MH109133 (National Institute of Mental Health) (R.D.M., J.M), R01GM123203 (National Institute of General Medical Sciences) (R.D.M.). We thank Tamara Abou-Antoun and Dr. Christian Shively for their assistance with western blots. We thank the Baldridge Lab at Washington University in Saint Louis for the generous use of their RT-qPCR machine.

\section{REFERENCES}

Aartsma-Rus, A., \& Krieg, A. M. (2017). FDA Approves Eteplirsen for Duchenne Muscular Dystrophy: The Next Chapter in the Eteplirsen Saga.Nucleic Acid Therapeutics, 27(1), 1-3. https://doi.org/10.1089/nat.2016.0657 Bilandzic, M., Rainczuk, A., Green, E., Fairweather, N., Jobling, T. W., Plebanski, M., \& Stephens, A. N. (2019). Keratin-14 (KRT14) Positive Leader Cells Mediate Mesothelial Clearance and Invasion by Ovarian Cancer Cells. Cancers, 11(9). https://doi.org/10.3390/cancers11091228 Cartegni, L., Wang, J., Zhu, Z., Zhang, M. Q., \& Krainer, A. R. (2003). ESEfinder: A web resource to identify exonic splicing enhancers.Nucleic Acids Research, 31(13), 3568-3571. Cong, L., Ran, F. A., Cox, D., Lin, S., Barretto, R., Habib, N., Hsu, P. D., Wu, X., Jiang, W., Marraffini, L. A., \& Zhang, F. (2013). Multiplex Genome Engineering Using CRISPR/Cas Systems. Science,339(6121), 819-823. https://doi.org/10.1126/science.1231143 Ekker, S. C. (2006). Morphants: A New Systematic Vertebrate Functional Genomics Approach. Yeast (Chichester, England), 17(4), 302-306. https://doi.org/10.1002/10970061(200012)17:4<302::AID-YEA53>3.0.CO;2-\# Geissler, R., Simkin, A., Floss, D., Patel, R., Fogarty, E. 
A., Scheller, J., \& Grimson, A. (2016). A widespread sequence-specific mRNA decay pathway mediated by hnRNPs A1 and A2/B1. Genes $\& 3$ Development, 30(9), 1070-1085. https://doi.org/10.1101/gad.277392.116 Gilbert, L. A., Horlbeck, M. A., Adamson, B., Villalta, J. E., Chen, Y., Whitehead, E. H., Guimaraes, C., Panning, B., Ploegh, H. L., Bassik, M. C., Qi, L. S., Kampmann, M., \& Weissman, J. S. (2014). Genome-Scale CRISPR-Mediated Control of Gene Repression and Activation. Cell,159(3), 647-661. https://doi.org/10.1016/j.cell.2014.09.029 Jain, N., Lin, H.-C., Morgan, C. E., Harris, M. E., \& Tolbert, B. S. (2017). Rules of RNA specificity of hnRNP A1 revealed by global and quantitative analysis of its affinity distribution. Proceedings of the National Academy of Sciences of the United States of America,114(9), 2206-2211. https://doi.org/10.1073/pnas.1616371114 Jean-Philippe, J., Paz, S., \& Caputi, M. (2013). hnRNP A1: The Swiss Army Knife of Gene Expression. International Journal of Molecular Sciences, 14 (9), 18999-19024. https://doi.org/10.3390/ijms140918999 Kampmann, M. (2018). CRISPRi and CRISPRa Screens in Mammalian Cells for Precision Biology and Medicine. ACS Chemical Biology,13(2), 406-416. https://doi.org/10.1021/acschembio.7b00657 Kashima, T., Rao, N., David, C. J., \& Manley, J. L. (2007). HnRNP A1 functions with specificity in repression of SMN2 exon 7 splicing. Human Molecular Genetics, 16(24), 3149-3159. https://doi.org/10.1093/hmg/ddm276 Koike-Yusa, H., Li, Y., Tan, E.-P., VelascoHerrera, M. D. C., \& Yusa, K. (2014). Genome-wide recessive genetic screening in mammalian cells with a lentiviral CRISPR-guide RNA library. Nature Biotechnology,32(3), 267-273. https://doi.org/10.1038/nbt.2800 Lee, Y., \& Rio, D. C. (2015). Mechanisms and Regulation of Alternative Pre-mRNA Splicing. Annual Review of Biochemistry, 84, 291-323. https://doi.org/10.1146/annurev-biochem-060614-034316 Mullenders, J., Fabius, A. W. M., Madiredjo, M., Bernards, R., \& Beijersbergen, R. L. (2009). A Large Scale shRNA Barcode Screen Identifies the Circadian Clock Component ARNTL as Putative Regulator of the p53 Tumor Suppressor Pathway. PLoS ONE, 4(3). https://doi.org/10.1371/journal.pone.0004798 Nogrady, B. (2019). The challenge of delivering RNA-interference therapeutics to their target cells. Nature, 574(7778), S8-S9. https://doi.org/10.1038/d41586-019-03071-9 Papafotiou, G., Paraskevopoulou, V., Vasilaki, E., Kanaki, Z., Paschalidis, N., \& Klinakis, A. (2016). KRT14 marks a subpopulation of bladder basal cells with pivotal role in regeneration and tumorigenesis.Nature Communications, 7(1), 11914. https://doi.org/10.1038/ncomms11914 Roberts, T. C., Langer, R., \& Wood, M. J. A. (2020). Advances in oligonucleotide drug delivery. Nature Reviews Drug Discovery,19(10), 673-694. https://doi.org/10.1038/s41573020-0075-7 Stein, C. A., \& Castanotto, D. (2017). FDA-Approved Oligonucleotide Therapies in 2017. Molecular Therapy, 25(5), 1069-1075. https://doi.org/10.1016/j.ymthe.2017.03.023 Tycko, J., Wainberg, M., Marinov, G. K., Ursu, O., Hess, G. T., Ego, B. K., Aradhana, Li, A., Truong, A., Trevino, A. E., Spees, K., Yao, D., Kaplow, I. M., Greenside, P. G., Morgens, D. W., Phanstiel, D. H., Snyder, M. P., Bintu, L., Greenleaf, W. J., .. . Bassik, M. C. (2019). Mitigation of off-target toxicity in CRISPR-Cas9 screens for essential non-coding elements. Nature Communications,10(1), 4063. https://doi.org/10.1038/s41467-019-11955-7 Zhu, L.-F., Hu, Y., Yang, C.-C., Xu, X.-H., Ning, T.-Y., Wang, Z.-L., Ye, J.-H., \& Liu, L.-K. (2012). Snail overexpression induces an epithelial to mesenchymal transition and cancer stem cell-like properties in SCC9 cells. Laboratory Investigation, 92(5), 744-752. https://doi.org/10.1038/labinvest.2012.8

\section{FIGURE LEGENDS}

Figure 1: Overview of hnRNPA1 recruiting molecules (AROs).(A) Each ARO consists of two parts, a 20 bp hnRNPA1 recruiting loop derived from HIV exon splicing silencer 3 , which recruits hnRNPA1 by binding to RNA binding domain RRM1, and an antisense targeting domain consisting of 20-25 base pairs of RNA complementary to a sequence on a target pre-mRNA. (B) The hypothesized mechanism of AROs. 1. In the absence of AROs splicing occurs normally. 2. The binding of an ARO to its pre-mRNA target recruits hnRNPA1 and displaces SR proteins and spliceosome machinery (U2, U2AF65/35, and U1). 3. We hypothesize this causes repression of splicing resulting in exon skipping or intron inclusion. This would cause frameshifts in the mRNA leading to the presence of premature termination codons (PTCs) in the reading frame upstream of exonic junction complexes (EJCs). UPF1 would then recognize these sites as mis-spliced mRNA and degrade the RNA by non-sense mediated decay.

Figure 2: Plasmid expressed AROs knock down KRT14 in human keratinocytes (HaCat cells). (A) Visual representation of each ARO and controls. 1. scrOligo-ARL is a scrambled targeting oligo with an intact 
hnRNPA1 recruiting loop (ARL). 2. Oligo is a targeting oligo with homology to Exon 7 of KRT14. 3. ARLOligo is the targeting oligo with an ARL on the 5' end of the construct relative to the targeting domain. 4. Oligo-ARL is the targeting oligo with an ARL on the 3 ' end of the construct relative to the targeting domain. 5. ARL-Oligo-ARL is a construct with ARLs attached to both the 5' and 3' ends of the targeting domain. (B) Fold change of KRT14 transcript expression relative to a carrier only lipofectamine control RT-qPCR. Cells were lipofected with plasmid for 48 hours and then harvested for RNA extraction. Green bars indicate plasmid experiments while blue bars indicate experiments with siRNA designed to knock down hnRNPA1. Three replicates were performed. Error bars show standard deviation between replicates. A Student's t test was used to determine significance.

Figure 3: Plasmid expressed AROs targeting exon 14 knock down TBK1 RNA expression in human keratinocytes (HaCat cells). All AROs were expressed with ARLs attached to the 3' and 5' ends of the targeting oligo. (A) Location of targeting oligo homology to exon 14 of TBK1. (B) Fold change of TBK1 transcript expression relative to a carrier only lipofectamine control RT-qPCR. Cells were lipofected with plasmid for 48 hours and then harvested for RNA extraction. Three replicates were performed. Error bars show standard deviation between replicates. A Student's t-test was used to determine significance.

Figure 4: Synthesized single-strand RNA oligonucleotides knock down KRT14 expression across two cell types. (A) Synthesized ssRNAs with phosphorothioate bonds on the first two and last two bases administered via lipofection to HaCat cells at varying concentrations. ARL-Oligo-ARL refers to a ssRNA with two hnRNPA1 recruiting loops on either side of the targeting domain. ARL-Oligo is composed of a single hnRNPA1 recruiting loop fused to the 5' end of an RNA targeting sequence. A previously validated siRNA against KRT14 was used as a positive control at each concentration. Cells were harvested 48 hours post lipofection and fold change was determined relative to a lipofectamine carrier only control. (B) The single ARL-Oligo ssRNA and siRNA targeting KRT14 were lipofected into SCC9 cells. As previously, cells were harvested 48 hours post lipofection and fold change was determined relative to a lipofectamine carrier only control. Three replicates were performed in each experiment. Error bars show standard deviation between replicates. A Student's t-test was used to determine significance.

Figure 5: AROs show a functional phenotype in a cancer invasion model. (A) As previously, siRNA targeting KRT14 and an ssRNA ARO with a single hnRNPA1 recruiting loop fused to the 5' end of a KRT14 targeting oligo were administered at $100 \mathrm{nM}$ via lipofection in SCC9 cells and grown for 48 hours. 20,000 cells were seeded inside a matrigel-coated transwell for a Boyden chamber invasion assay and allowed to migrate through the pores to the other side of the membrane for 24 hours. Cells were then stained with crystal violet and invading cells were imaged and counted. Two replicates were preformed of this experiment. Error bars show standard deviation between replicates. A Student's t test was used to determine significance. (B)Representative images of crystal violet stained invading cells in each condition. (C) Protein abundance of KRT14 in cells lipofected with either ARO (ssRNA ARL-Oligo) or anti-KRT14 siRNA at a final concentration of $100 \mathrm{nM}$. Each condition was performed in triplicate and total protein was harvested 48 hours after lipofection. Western blots were preformed using KRT14 mouse primary antibody and an anti-mouse HRP conjugated secondary antibody, and total protein load using a stain free gel was used as a loading control for normalization. 
A.

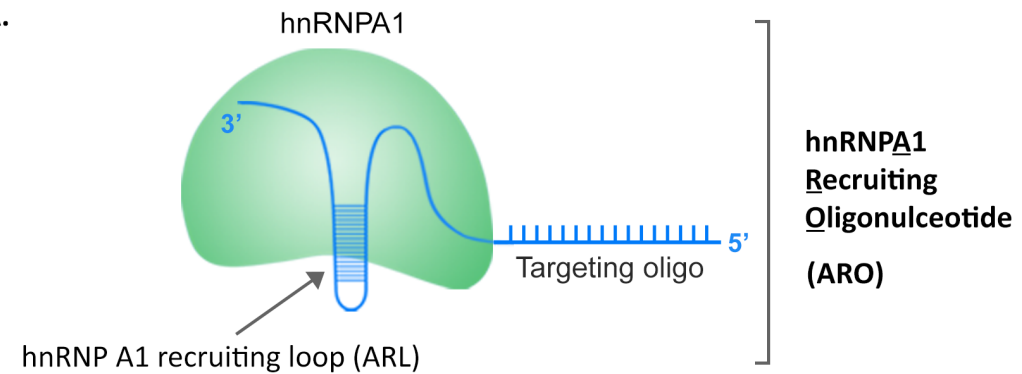

B.

Hypothesized ARO mechanism

1. Normal splicing occurs in the absence of AROs

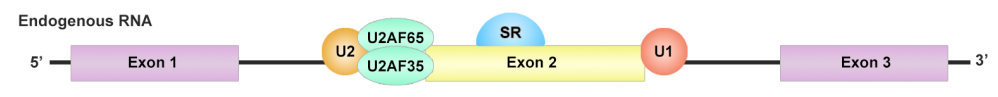

2. ARO recruits hnRNPA1 disrupting splicing machinery

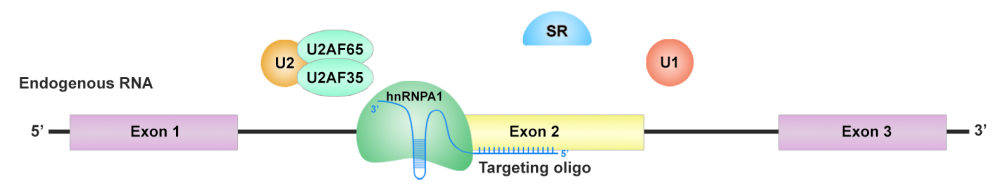

3. Splice site discruption destabilizes target mRNA leading to degradation

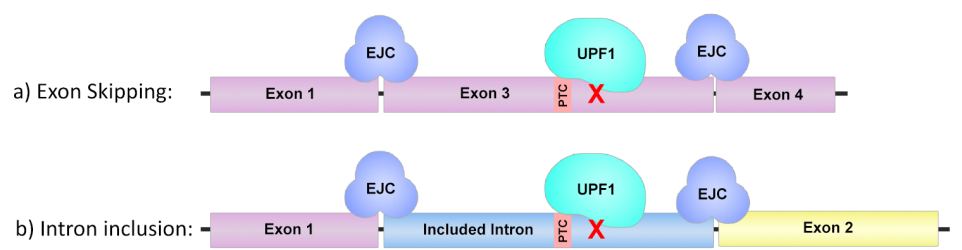


A.

Visual represenatiation of AROs and controls.

1. scrambledOligo-ARL

2. Oligo
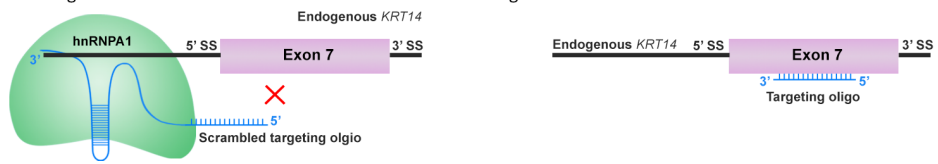

3. ARL-Oligo

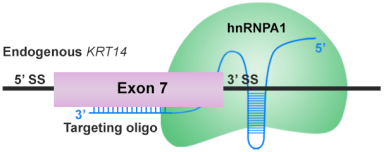

4. Oligo-ARL
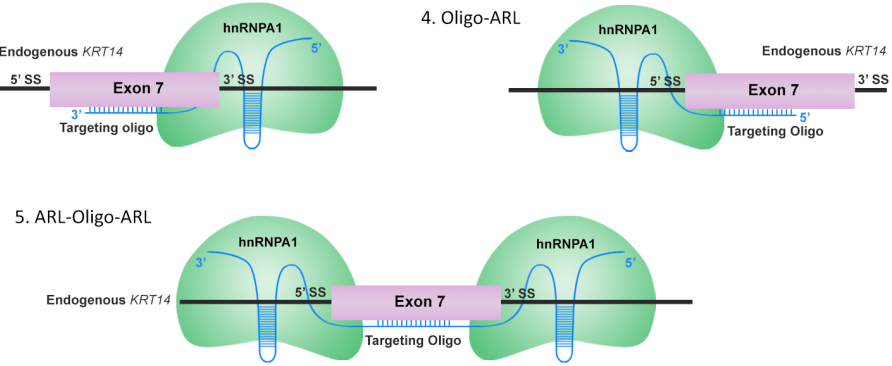

B.

KRT14 Expression In HaCat Cells

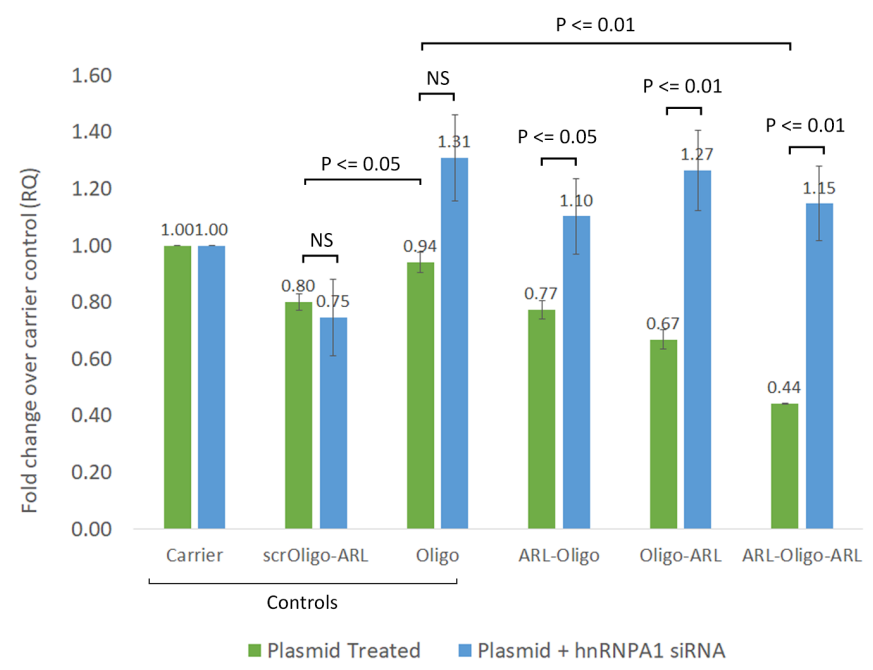


A.

Location of targeting olgionucleotides on TBK1

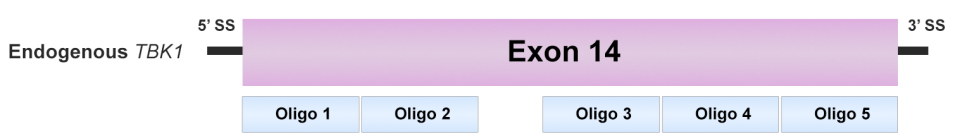

B.

TBK1 Expression In HaCat Cells

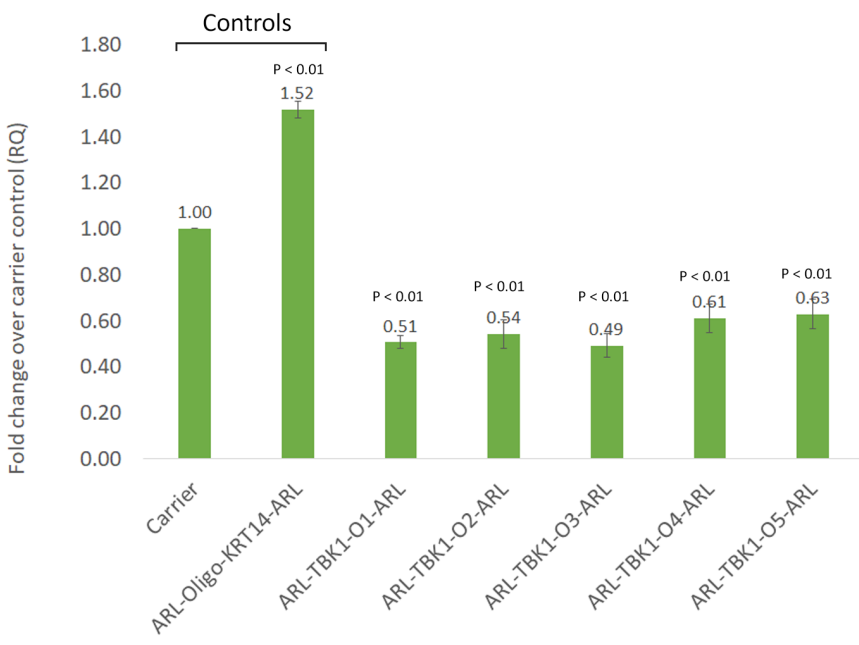


A.

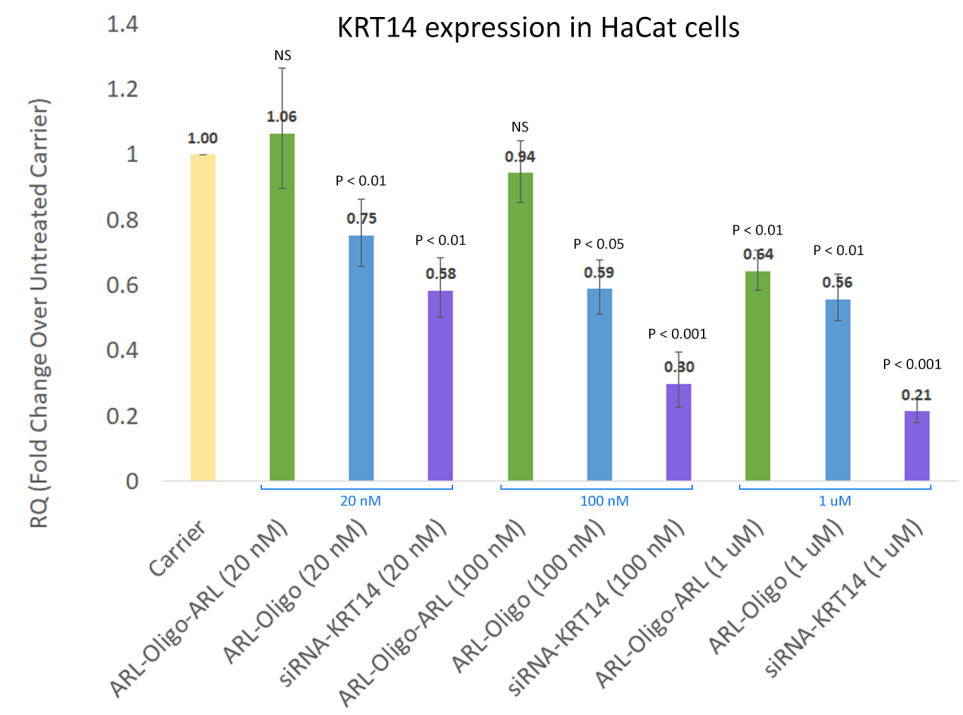

B.

1.20

KRT14 expression in SCC9 cells

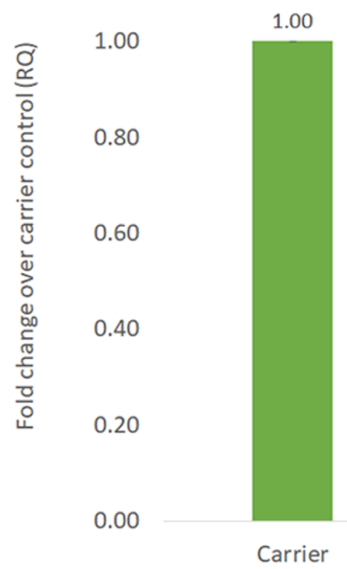

$\mathrm{P}<=0.001$

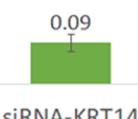

$\mathrm{P}<=0.001$

siRNA-KRT14

$\frac{<.01}{190}$ 
A.

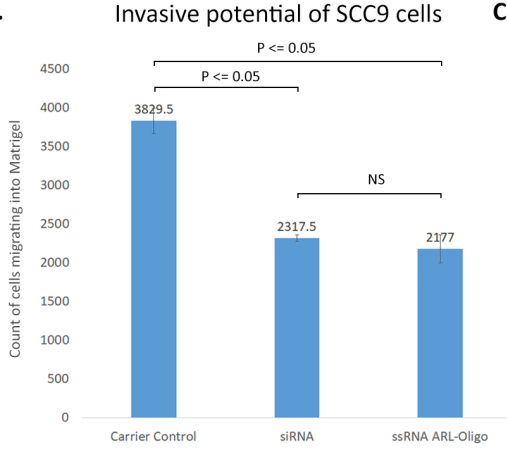

C. KRT14 protien expression in SCC9 cells 1.20

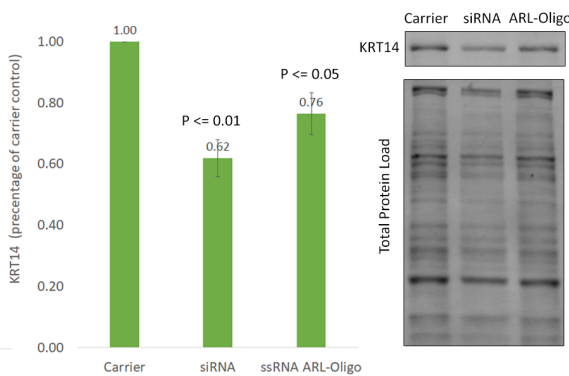

B.

SCC9 cell migration into transwells

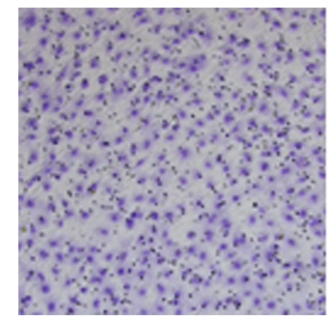

Carrier

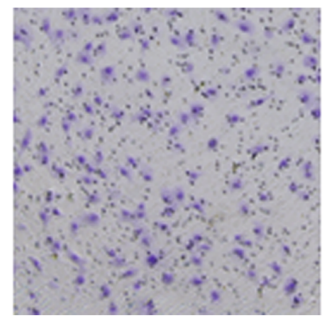

siRNA-KRT14

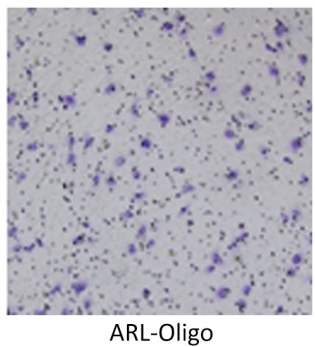

